\title{
Acetyl-l-carnitine prevents homocysteine-induced suppression of Nrf2/Keap1 mediated antioxidation in human lens epithelial cells
}

\author{
SHUI-PING YANG ${ }^{1}$, XIU-ZHEN YANG ${ }^{2}$ and GUO-PING CAO ${ }^{1}$ \\ Departments of ${ }^{1}$ Ophthalmology and ${ }^{2}$ Hepatic Disease, Taizhou People's Hospital, Taizhou, Jiangsu 225499, P.R. China
}

Received March 28, 2014; Accepted January 29, 2015

DOI: $10.3892 / \mathrm{mmr} .2015 .3490$

\begin{abstract}
Previous studies have revealed that high levels of serum homocysteine (Hcy) are closely associated with the development of juvenile and age-related cataracts. An increased concentration of Hcy is likely to induce gene specific demethylation in DNA promoter regions. The aim of the present study was to prevent this demethylation by administering acetyl-1-carnitine (ALCAR) to human lens epithelial cells (HLECs). Different concentrations of Hcy were used to treat HLECs for 3, 6, 12 and $24 \mathrm{~h}$ and the findings were used to determine the optimum dose to induce endoplasmic reticulum (ER) stress. Similarly, the concentration of ALCAR was standardized. The production of reactive oxygen species (ROS) and the percentage of cells undergoing cell death were measured. The levels of antioxidants, ER stress-associated proteins, mRNA levels of nuclear factor erythroid-2-related factor 2 (Nrf2), Kelch-like ECH-associated protein 1 (Keap1) and promoter DNA methylation of the Keapl gene were also assessed. Hcy was observed to induce ER stress, produce ROS and lead to cell death. However, administration of ALCAR prevented these effects to a significant degree. Additionally, western blot analysis revealed that ALCAR increased the levels of antioxidant proteins, including catalase, superoxide dismutase, glutathione peroxidase, Nrf2, Keap1 and glutathione. Similarly, the reverse transcription-quantitative polymerase chain reaction experiments on Nrf2 and Keap1, as well as the bisulfite genomic DNA sequencing analysis revealed a preventive effect of ALCAR against Hcy-induced ER stress. The ER stress-induced activation of the unfolded protein response is responsible for demethylation of Keap1 promoter DNA to activate the expression of the Keap1 protein, which then increases the targeting of Nrf2 for proteosomal degradation. This decrease in Nrf2 activity represses the tran-
\end{abstract}

Correspondence to: Dr Shui-Ping Yang, Department of Ophthalmology Taizhou People's Hospital, 210 Yangchun Road, Taizhou, Jiangsu 225499, P.R. China

E-mail: shuipingyang64@gmail.com

Key words: human lens epithelial cells, antioxidant, homocysteine, acetyl-1-carnitine, nuclear factor erythroid-2-related factor 2, DNA demethylation scription of numerous antioxidant enzyme genes and alters the redox-balance towards lens oxidation. However, treatment with ALCAR led to significant protection from these effects. The present results suggested that ALCAR either prevents or ameliorates the actions of the antioxidant system in HLECs at the level of the protein and the gene. Further advanced studies are required for the development of ALCAR as an anti-cataract agent.

\section{Introduction}

An increased level of serum homocysteine (Hcy) is closely associated with the development of juvenile cataracts and age-related cataracts (ARCs). Recent studies have demonstrated statistically significant increases in Hcy and decreases in vitamin B2, B6, B12 and folic acid in the plasma of 40 adult patients with ARCs (1). Additionally, a total of 24 of 29 children with homocystinuria type 1 were also diagnosed with cataracts (2). Homocystinuria in adults has been revealed to be acquired through chronic kidney disease, cigarette smoking and alcoholism in addition to dietary deficiencies of vitamin B2, B6, B12 and folic acid (3). Hcy is biosynthesized from methionine in a multistep process $(4,5)$. The co-enzymes involved in this process are folic acid and the vitamins B2, B6 and B12. Under the conditions of normal metabolism, the formation and elimination of Hcy is balanced. Under higher levels of Hcy, misfolded proteins have been observed to accumulate in the endoplasmic reticulum (ER), which can then induce the unfolded protein response (UPR) $(6,7)$.

Recent studies have found the cataractogenic stressors, including hypoxia with low glucose (8) or high glucose (9), Hcy (10) and galactose (9), induce ER stress-mediated activation of the UPR and overproduction of reactive oxygen species (ROS), resulting in death of lens epithelial cells (LECs). The ROS that have been generated then decrease the levels of cytosolic glutathione and contribute to an additional source of ROS from the mitochondria (11), which leads to cell death. In addition, Hcy was also reported to suppress nuclear factor erythroid-2-related factor 2 (Nrf2; also termed as NFE2L2) dependent antioxidant protection leading to the overproduction of ROS in LECs $(8,10)$. Nrf2 leads to the transcription of 200 protective genes, including 20 enzymatic antioxidant genes $(12,13)$. Recently, Gao et al (14) reported promoter DNA demethylation of Kelch-like ECH-associated protein 1 (Keap1), which is a negative regulator of Nrf2, in human lens 
epithelial cells (HLECs) and in age-related cataractous lenses. These sequential events may be responsible for aging and cataract formation in humans.

Acetyl-L-carnitine ( $\gamma$-trimethyl- $\beta$-acetyl-butyrrobetaine) is the acetyl ester of the trimethylated amino acid L-cartinine. Carnitine and its short-chain esters facilitate transport of long-chain fatty acids across the inner mitochondrial membrane for $\beta$-oxidation, thereby promoting energy availability and preventing toxic accumulation of long-chain fatty acids (15). The enzyme carnitine acetyltransferase catalyses the formation of acetyl-L-carnitine from carnitine and acetyl-coenzyme A (acetyl-CoA) and also the reversible reaction. The modulation of the intracellular concentration of free CoA and acetyl-CoA has been established to be a common mechanism for the various physiological activities of acetyl-L-carnitine, such as the acetylation of $\mathrm{H} 4$ histones $(15,16)$. It has been reported that ALCAR may be a promising candidate for arresting Hcy-induced Alzheimer disease-like pathological and behavioral impairments (17). In addition, ALCAR has been previously revealed to exhibit an anti-cataractogenic effect in against selenite-injection and buthionine sulfoximine (BSO)-injection rat models $(18,19)$. The aim of the present study was to investigate the efficacy of ALCAR in the protection against the adverse effect of Hcy in HLECs.

\section{Materials and methods}

HLEC culture. HLECs (HLEC-SRA 01/04; Clonetics, Walkersville, MD, USA) were cultured overnight in Dulbecco's modified Eagle's medium (DMEM) high glucose (Gibco Life Technologies, Carlsbad, CA, USA) supplemented with $10 \%$ fetal bovine serum under $20 \%$ atmospheric oxygen at $37^{\circ} \mathrm{C}$. The cells were harvested and used for the measurement of ROS levels, cell death analyses, western blotting, reverse transcription-quantitative polymerase chain reaction (RT-qPCR) and bisulfite genomic DNA sequencing.

Measurement of ROS and cell death. Using fluorescence-activated cell sorting (FACSDiva v4.0.1; BD Biosciences, Sydney, Australia), ROS production and cell death rates were measured. Cells were plated $24 \mathrm{~h}$ prior to the experiment, maintained in DMEM low glucose (Gibco Life Technologies) and cultured with different concentrations of Hcy $(50,75,100$ or $125 \mu \mathrm{M})$ or acetyl-1-carnitine $(50,100$ or $150 \mu \mathrm{M})$ for 6,12 and $24 \mathrm{~h}$ in order to determine the optimum concentration and time period for experimentation. Further experiments were then performed for $24 \mathrm{~h}$ with the determined optimum dosage.

Western blot analysis. At the end of experimental treatments, HLECs were harvested, washed with ice-cold phosphate-buffered saline (PBS) and lysed with radioimmunoprecipitation assay buffer (Cell Signaling Technology, Danvers, MA, USA). The lysates were centrifuged at $13,000 \mathrm{x}$ g for $10 \mathrm{~min}$ and the protein content of the supernatant was determined using the Bradford method (20). Soluble proteins (10-20 $\mu \mathrm{g})$ were loaded and separated by 10\% SDS-PAGE and blotted onto a polyvinylidene fluoride membrane (Sigma-Aldrich, St. Louis, MO, USA). Subsequently, the membranes were blocked with 5\% non-fat dry milk powder solution for $1 \mathrm{~h}$ at room temperature prior to an overnight incubation with the following primary antibodies (1:1,000 dilution): Rabbit polyclonal anti-catalase (CAT; cat. no. NBP2-24916; Novus Biologicals, LLC, Littleton, CA, USA), rabbit polyclonal anti-glutathione reductase (GR; cat. no. NBP2-24940; Novus Biologicals, LLC), rabbit polyclonal anti-glutathione-s-transferase (GST; cat. no. NBP2-16686; Novus Biologicals, LLC), mouse monoclonal anti-immunoglobulin heavy-chain binding protein (BiP; cat. no. 610978; BD Biosciences), rabbit polyclonal anti-inositol-requiring enzyme $1 \alpha$ (IRE1 $\alpha$; cat. no. PA5-20189; Thermo Fisher Scientific, Waltham, MA, USA), rabbit polyclonal anti-activating transcription factor 6 (ATF6; cat. no. PA5-20215; Thermo Fisher Scientific), rabbit polyclonal anti-Nrf2 (cat. no. PA5-27882; Thermo Fisher Scientific) and goat polyclonal Keap1 (cat. no. sc-15246; Santa Cruz Biotechnology, Inc., Santa Cruz, CA, USA) at $4^{\circ} \mathrm{C}$. Following rinsing of the membranes with Tris-buffered saline with Tween 20, they were incubated with the secondary antibody (1:5,000 dilution) for $1 \mathrm{~h}$ at room temperature and the bands were visualized using enhanced chemiluminescence as described previously (10). The intensity of each band was normalized to that of $\beta$-actin and quantified using ImageJ version 1.48 software (National Institutes of Health, Bethesda, MD, USA).

RT-qPCR analysis. Total RNA was extracted from the HLECs with Quick-RNA MicroPrep solution (Zymo Research Corporation, Orange, CA, USA) according to the manufacturer's instructions. Subsequently, the purified total RNA was reverse-transcribed using iScript reverse transcription supermix for real-time PCR (Bio-Rad, Hercules, CA, USA) according to the manufacturer's instructions. The reverse transcribed RNA was analyzed using qPCR using the SsoFast EvaGreen supermix (Bio-Rad). The optimal qPCR assay for Nrf2 and Keap1 genes was performed according to Roche's ProbeFinder (http://qper. probefinder.com/organism.jsp). The primers were purchased from Generay (Shanghai) Biotech Co., Ltd. (Shanghai, China). The primer sequences were as follows: Forward: 5'-ACACGGTCCACAGCTCATC-3' and reverse: 5'-TGCCTC CAAAGTATGTCAATCA-3' for Nrf2, with a product size of 96 bp; forward: 5'-GGGTCCCCTACAGCCAAG-3' and reverse: 5'-TGG GGTTCCAGAAGATAAGC-3' for Keap1, with a product size of $66 \mathrm{bp}$; and forward: 5'-CCAACCGCGAGA AGATGA-3' andreverse:5'-CCAGAGGCGTACAGGGATAG-3' for $\beta$-actin, with a product size of $97 \mathrm{bp}$. Each reaction was conducted in triplicate and a standard curve was prepared using a serial dilution of a reference sample. The relative copy numbers were obtained from the standard curve and were normalized to the values obtained for $\beta$-actin as the internal control. The results of the PCR were quantified using the $2^{-\Delta \Delta C T}(21)$.

Bisulfite conversion and DNA sequencing. The genomic DNA of HLECs was subjected to bisulfite conversion using the EZ DNA Methylation-Direct ${ }^{\mathrm{TM}}$ kit (Zymo Research Corporation). The bisulfite converted DNA was then used for bisulfite genomic DNA sequencing. The bisulfite-modified DNA was amplified via bisulfite sequencing PCR using Platinum PCR SuperMix High Fidelity (Invitrogen Life Technologies, Carlsbad, CA, USA) with primers specific to the human Keap1 promoter region $(-430$ to -110$)$ with a product of $330 \mathrm{bp}$. The sequences were as follows: forward: 5'-TTAGTTATTTAGGAGGTTGT-3' and 

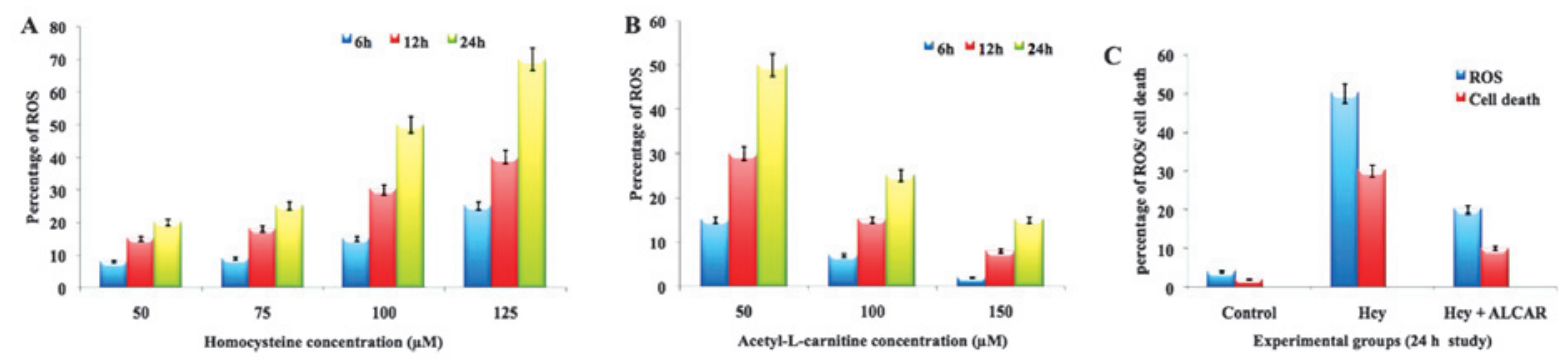

Figure 1. Dosage determination. (A) Percentage of ROS production in human lens epithelial cells exposed to different concentrations $(50,75,100$ and $125 \mu \mathrm{M})$ of homocysteine. (B) Cells exposed to homocystine $(100 \mu \mathrm{M})$ and various concentrations of ALCAR $(50,100$ or $150 \mu \mathrm{M})$. (C) Percentage of ROS production and cell death measured in the experimental groups. ROS, reactive oxygen species.

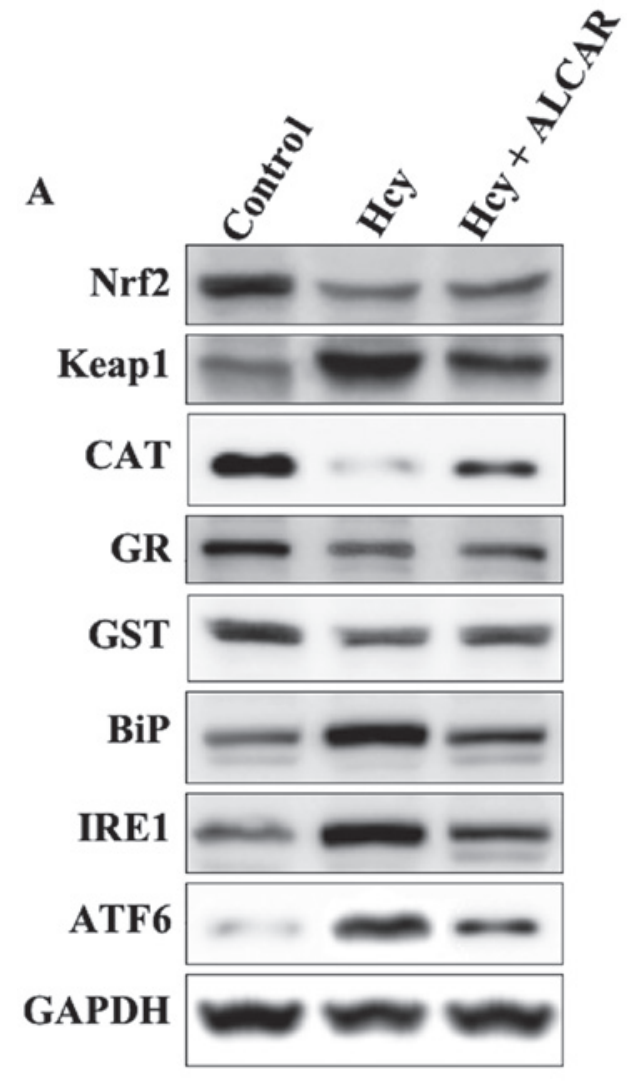

B

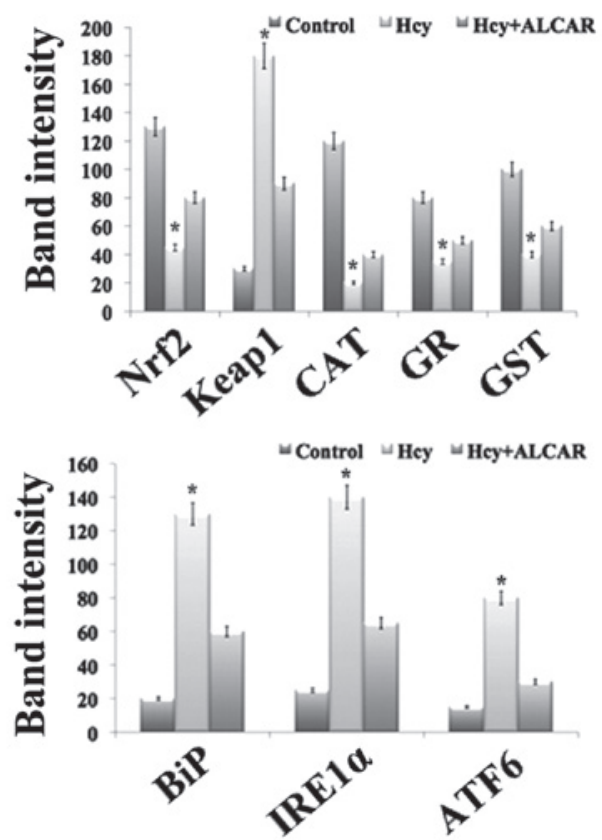

Figure 2. Results of western blot analysis. (A) Representative western blot analysis of the Nrf2/Keap1 mediated antioxidant system and endoplasmic reticulum stress-associated proteins in human lens epithelial cells of the experimental groups: Control, Hcy alone, and Hcy and ALCAR in combination at a concentration of $100 \mu \mathrm{M}$ each. (B) Representive quantification of band intensity of the corresponding western blots. " $\mathrm{P}<0.05$, compared with control and Hcy+ALCAR treated group. Keap1, Kelch-like ECH-associated protein 1; Hcy, homocysteine; ALCAR, acetyl-1-carnitine; Nrf2, nuclear factor erythroid-2-related factor 2; CAT, catalase; GR, glutathione reductase; GST, glutathione-s-transferase; BiP, immunoglobulin heavy-chain binding protein; IRE1, inositol-requiring enzyme 1; ATF6, activating transcription factor 6 .

reverse: 5'-AACCCCCCTTCTCACTA-3'. The primers were designed using the Methyl Primer Express v1.0 software from Applied Biosystems (Foster City, CA, USA). The PCR products were purified by gel extraction using the Zymoclean ${ }^{\text {TM }}$ Gel DNA recovery kit (Zymo Research Corporation) and then cloned into $\mathrm{pCR} 4-\mathrm{TOPO}$ vectors using the TOPO TA Cloning kit (Invitrogen Life Technologies). The recombinant plasmids were transformed into One Shot TOP10 chemically competent Escherichia coli (Invitrogen Life Technologies) using the regular chemical transformation method as described in the manufacturer's instructions. Plasmid DNA was prepared from $\sim 12$ independent clones of each amplicon with the PureLink
Quick Plasmid Miniprep kit (Invitrogen Life Technologies) and sequenced to determine the status of $\mathrm{CpG}$ methylation. Subsequently, the sequenced data of each clone was analyzed for DNA methylation in the Keap1 promoter using BISMA software (http://biochem.jacobs-university.de/BDPC/BISMA/) using the default filtering threshold settings (22).

Statistical analysis. The results were expressed as the mean \pm standard deviation, and statistical significance was evaluated by Student's t-test using SPSS version 15.0 (SPSS Inc., Chicago, IL, USA). $\mathrm{P}<0.05$ was considered to indicate a statistically significant difference. 


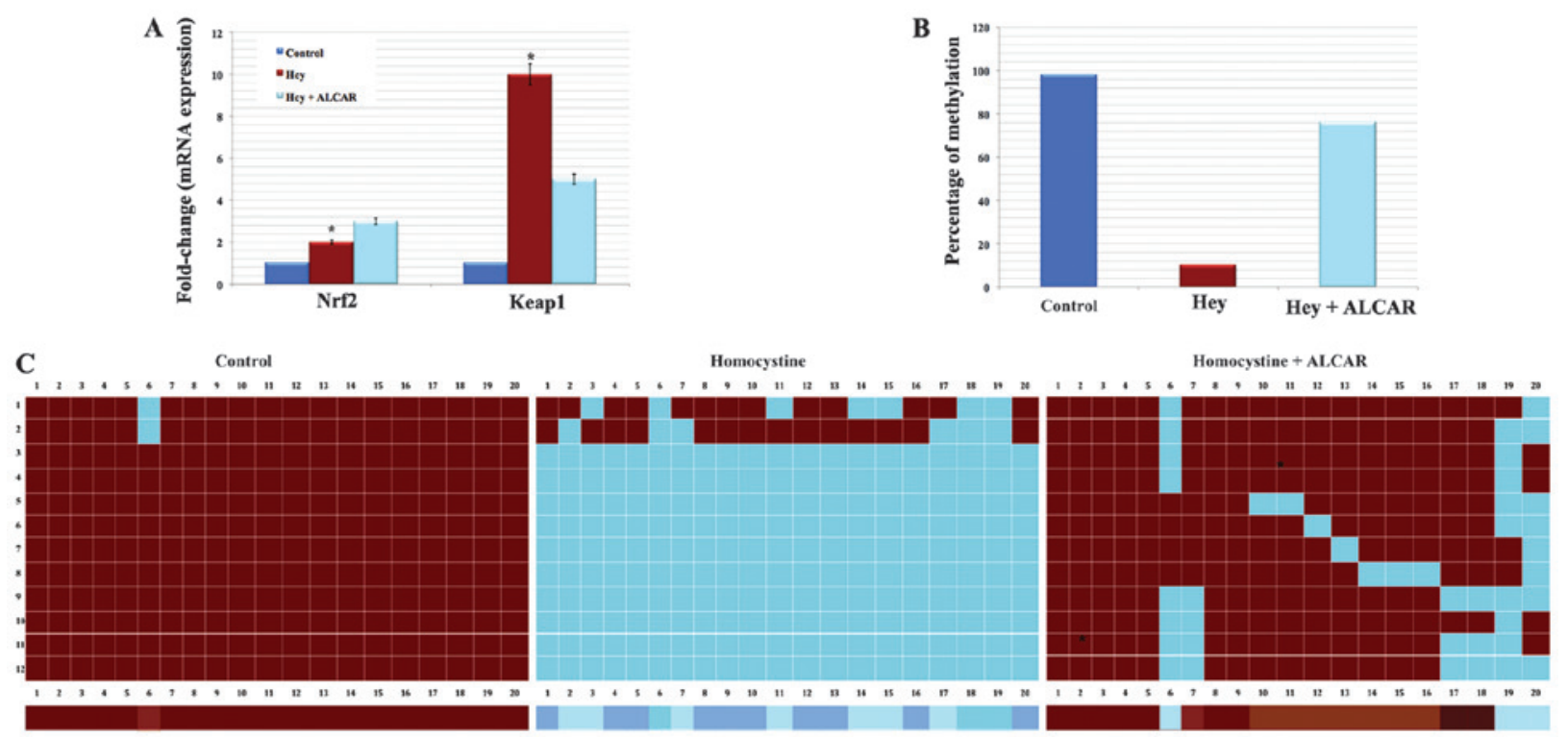

Figure 3. Protective effect of ALCAR in gene expression and promoter DNA demethylation. (A) Representative of the reverse transcription-quantitative polymerase chain reaction results demonstrating the mRNA levels of the Nrf2 and Keap1 gene in the experimental groups. "P $<0.05$, compared with control and Hcy+ALCAR treated group. (B) Representative percentage of demethylation based on the sequencing results. (C) Methylation pattern in the Keap1 promoter region between -430 and -110 of control HLECs exhibit highly methylated CpG dinucleotides, Hcy-treated cells exhibit highly demethylated CpG dinucleotides and HLECs treated with Hcy and ALCAR exhibit moderately demethylated CpG dinucleotides. Red colored boxes denote methylcytosine and blue colored boxes denote unmethylated cytosine, respectively. The color gradient bar, which represents the red region, has more methylated CpGs and the blue region has more unmethylated CpGs. Keap1, Kelch-like ECH-associated protein 1; Hcy, homocysteine; ALCAR, acetyl-1-carnitine; Nrf2, nuclear factor erythroid-2-related factor 2; HLECs, human lens epithelial cells.

\section{Results}

Dosage determination. The optimum concentration was obtained by administering various concentrations of Hcy (50, 75, 100 and $125 \mu \mathrm{M})$ and ALCAR (50, 100 and $150 \mu \mathrm{M})$ in HLECs at different time intervals, including 6,12 and $24 \mathrm{~h}$. The dosage of Hcy was determined by the level of ROS production and cell death. Similarly, the dosage of ALCAR was determined by its protective effects against ROS and cell death resulting from Hcy exposure. The Hcy concentration of $100 \mu \mathrm{M}$ exhibited a low level of ROS production from $6 \mathrm{~h}$ and increased considerably at $24 \mathrm{~h}$ (Fig. 1A). Whereas concentrations of 50 and 75 exhibited a low level of ROS production and $125 \mu \mathrm{M}$ demonstrated a high level of ROS production and cell death at $24 \mathrm{~h}$. ALCAR exhibited a greater protective effect at a concentration of $150 \mu \mathrm{M}$, as compared with the other concentrations assessed (Fig. 1B). Considering these results, the experiment was performed for $24 \mathrm{~h}$ with the optimum concentration of Hcy $(100 \mu \mathrm{M})$ and ALCAR $(100 \mu \mathrm{M})$, from which ALCAR exhibited a significant protective effect against Hcy in HLECs (Fig. 1C).

ALCAR increases the levels of antioxidant proteins and decreases the levels of ER stress-associated proteins. Following the dosage determination, the antioxidant and ER stress-associated protein levels in experimental groups, including control, Hcy alone and Hcy with ALCAR were investigated. Western blotting analysis of the Nrf2/Keap1-dependent antioxidant protection system in HLECs treated with $100 \mu \mathrm{M}$ Hcy for $24 \mathrm{~h}$ was performed as Nrf2 is a nuclear transcriptional factor, which controls $>200$ stress-associated genes and Keap1 is a negative regulator of the Nrf2 protein. Notably, the protein levels of Nrf2 and its downstream enzymes, including GR, GST and CAT were significantly decreased in HLECs treated with $100 \mu \mathrm{M}$ Hcy for $24 \mathrm{~h}$, with a marked increase in the level of Keap1 (Fig. 2). However cells treated with Hcy and ALCAR $(100 \mu \mathrm{M})$ exhibited a significantly increased level of Nrf2 and its downstream antioxidant proteins. Notably, ALCAR had a significant effect on the level of Keap1 protein. Similarly, Hcy induced an increase in the ER stress-associated proteins, BiP, ATF6 and IRE1 $\alpha$, which were also inhibited in the presence of ALCAR (Fig. 2). These results suggested that Hcy induced suppression of Nrf2/Keap1-dependent antioxidant protection through ER stress mediated ROS production and this was inhibited by treatment with ALCAR.

ALCAR treatment increases Nrf2/Keap1 mRNA levels. Following the protein blot results, subsequent quantification of the mRNA levels of expression of Nrf2 and Keap1 genes in the experimental groups was conducted. RT-qPCR was performed to analyze or quantify the mRNA transcript level. Notably, these results reflected the protein immunoblot results. A decrease in the level of Nrf2 and significant increase in Keap1 mRNA levels in the Hcy exposed cells was observed. However, these changes in mRNA levels were inhibited by ALCAR treatment (Fig. 3A). These results confirmed that Hcy mediated Keap1 promoter DNA demethylation, which significantly increased Keap1 transcription and this is controlled by ALCAR treatment.

ALCAR prevents Hcy-induced DNA demethylation. Bisulphite genomic DNA sequencing of the Keap1 promoter region (20 CpGs; between -430 to -110) was performed in HLECs of the experimental groups to investigate whether the marked 
Keap1 gene expression may be due to demethylation. The normal HLECs (control) exhibited $100 \%$ methylation of Keap1 promoter DNA. Notably, HLECs treated with Hcy alone exhibited $\sim 90 \%$ loss of 5-methylcytosine in the Keap1 promoter region than that of the control HLECs (Fig. 3B and C). However, HLECs exposed to Hcy and ALCAR exhibited $\sim 80 \%$ methylation. The present results suggested that loss of Keap1 promoter DNA methylation eventually leads to overexpression of Keap1 resulting in Nrf2 suppression, as Keap1 is a negative regulator of Nrf2. Thus, it was hypothesized that ALCAR prevented the Hcy induced promoter DNA demethylation of Keap1 (Fig. 3B and C).

\section{Discussion}

The present study demonstrated that Hcy induces ER stress-mediated UPR activation, ROS overproduction and suppression of Nrf2/Keap1 dependent antioxidant protection by Keap1 promoter demethylation, leading to cell death in HLECs. These sequential events may be a critical mechanism for cataract formation. However, it is hypothesized that ALCAR may protect against Hcy-induced adverse effects in HLECs, thereby preventing the formation of cataracts. Previously, Elanchezhian et al (18) demonstrated the protective effect of ALCAR in selenite-induced cataractogenesis in a rat model. Considering the potential of ALCAR treatment, the present study was designed in HLECs against the adverse effect of Hcy. A high level of serum Hcy is closely associated with juvenile and age-associated cataracts. Recent studies have revealed statistically significant increases of Hcy and decreases in vitamins B2, B6, B12 and folic acid in the plasma of 40 adult patients with ARCs (1). In addition, 24 of 29 children with homocystinuria type 1 were also diagnosed with cataracts (2). Consistent with these studies, the present study revealed that HLECs exposed to $100 \mu \mathrm{M}$ Hcy for $24 \mathrm{~h}$ was capable of inducing the production of ROS and cell death.

In addition, the induction of ER stress mediated UPR activation by various ER stressors, such as Hcy (10), tunicamycin (9), galactose (9) and hypoxia with low or high glucose $(8,9)$, eventually results in UPR activation, ROS overproduction and cell death of LECs. In the present study, the immunoblot analysis of Nrf2 mediated antioxidant proteins were observed to be decreased and ER stress-associated proteins were found to be increased in Hcy treated cells. Following treatment with ALCAR, these changes were prevented. A similar effect was observed in Nrf2 and Keap1 gene expression. The action of ALCAR in protecting the expression of genes involved in apoptosis suggested an additional pathway for its effect in retarding selenite cataractogenesis by protecting against the abnormal expression of genes involved in apoptosis and of antioxidant genes (23). Administration of ALCAR was observed to prevent the decreased expression of calpain Lp82 proteins and decreased levels of m-calpain mRNA transcripts in selenite-induced cataractogenesis (19), again suggesting the anti-apoptotic effect of ALCAR.

It has been well-established that the development of ARCs is tightly coupled with lens oxidation and aging $(24,25)$. The present results are concomitant with the previous findings, as ER stress induced by Hcy establishes production of ROS and suppresses the Nrf2/Keap1 mediated antioxidant system, promoting DNA demethylation in the Keap1 promoter region. The promoter DNA demethylation of the Keap1 gene resulted in the overexpression of the Keap1 protein and, as a result, the Nrf2 levels were ultimately decreased through proteasomal degradation. Thereby, Nrf2/Keap1 mediated antioxidant protection deteriorated and the cellular redox balance in the HLECs were shifted towards lens oxidation leading to cataract formation. Furthermore, aging is associated with a gradual progression of epigenetic promoter DNA demethylation of the Keap1 gene in human lenses, whereas, ER stressors are associated with rapid, radical and severe loss of Keap1 promoter demethylation leading to ARC formation (14). The treatment of ALCAR was observed to significantly protect the cells from these effects of Hcy. It has been reported that ALCAR may be a promising candidate for arresting Hcy-induced Alzheimer disease-like pathological and behavioral impairments (17). In addition, ALCAR has been previously revealed to exhibit an anti-cataractogenic effect in rat models against selenite-injection and BSO-injection $(18,19)$. The present investigation further demonstrated its protective effect in HLECs.

In conclusion, the present study identified that simultaneous supplementation of exogenous ALCAR significantly rescued HLECs from Hcy-induced ER stress and DNA demethylation of the Keapl gene. ALCAR controlled the production of ROS, protein levels of antioxidants, ER stress signaling, and mRNA levels of Nrf2 and Keap1 gene in HLECs from Hcy mediated adverse effects. The present findings suggested that ALCAR is a potential component in protecting the human lens from oxidative stress-induced damage, thereby preventing the formation of ARCs.

\section{References}

1. Sen SK, Pukazhvanthen P and Abraham R: Plasma homocysteine, folate and vitamin $\mathrm{B}(12)$ levels in senile cataract. Indian J Clin Biochem 23: 255-257, 2008.

2. Sulochana KN, Amirthalakshmi S, Vasanthi SB, et al: Homocystinuria with congenital/developmental cataract. Indian J Pediatr 67: 725-728, 2000.

3. Cumurcu T, Sahin S and Aydin E: Serum homocysteine, vitamin B 12 and folic acid levels in different types of glaucoma. BMC Ophthalmol 6: 6, 2006.

4. Selhub J: Homocysteine metabolism. Annu Rev Nutr 19: 217-246, 1999.

5. Williams KT and Schalinske KL: Homocysteine metabolism and its relation to health and disease. Biofactors 36: 19-24, 2010.

6. Ron D and Walter P: Signal integration in the endoplasmic reticulum unfolded protein response. Nat Rev Mol Cell Biol 8: 519-529, 2007.

7. Todd DJ, Lee AH and Glimcher LH: The endoplasmic reticulum stress response in immunity and autoimmunity. Nat Rev Immunol 8: 663-674, 2008.

8. Elanchezhian R, Palsamy P, Madson CJ, et al: Low glucose under hypoxic conditions induces unfolded protein response and produces reactive oxygen species in lens epithelial cells. Cell Death Dis 3: e301, 2012.

9. Ikesugi K, Yamamoto R, Mulhern ML and Shinohara T: Role of the unfolded protein response (UPR) in cataract formation. Exp Eye Res 83, 508-516, 2006.

10. Elanchezhian R, Palsamy P, Madson CJ, Lynch DW and Shinohara T: Age-related cataracts: homocysteine coupled endoplasmic reticulum stress and suppression of Nrf2-dependent antioxidant protection. Chem Biol Interact 200: 1-10, 2012.

11. Huang HC, Nguyen T and Pickett CB: Phosphorylation of Nrf2 at Ser-40 by protein kinase $\mathrm{C}$ regulates antioxidant response element-mediated transcription. J Biol Chem 277: 42769-42774, 2002. 
12. Enomoto A, Itoh K, Nagayoshi E, et al: High sensitivity of Nrf2 knockout mice to acetaminophen hepatotoxicity associated with decreased expression of ARE-regulated drug metabolizing enzymes and antioxidant genes. Toxicol Sci 59: 169-177, 2001.

13. Kaspar JW, Niture SK and Jaiswal AK: Nrf2:INrf2 (Keap1) signaling in oxidative stress. Free Radic Biol Med 47: 1304-1309, 2009.

14. Gao Y, Yan Y and Huang T: Human age-related cataracts: Epigenetic suppression of the nuclear factor erythroid 2-related factor 2-mediated antioxidant system. Mol Med Rep 11: 1442-1447, 2015.

15. Peluso G, Petillo O, Barbarisi A, et al: Carnitine protects the molecular chaperone activity of lens alpha-crystallin and decreases the protein post-translational modifications induced by oxidative stress. FASEB J 15: 1604-1606, 2001.

16. Riccioloni R, Scalibastri M, Kelleher JK, et al: Role of acetyl-L-carnitine in rat brain lipogenesis: implications for polyunsaturated fatty acid biosynthesis. J Neurochem 71: 2510-2517, 1998.

17. Zhou P, Chen Z, Zhao N, et al: Acetyl-L-carnitine attenuates homocysteine-induced Alzheimer-like histopathological and behavioral abnormalities. Rejuvenation Res 14: 669-679, 2011.

18. Elanchezhian R, Ramesh E, Sakthivel M, et al: Acetyl-L-carnitine prevents selenite-induced cataractogenesis in an experimental animal model. Curr Eye Res 32: 961-971, 2007.
19. Elanchezhian R, Sakthivel M, Geraldine P and Thomas PA: The effect of acetyl-L-carnitine on lenticular calpain activity in prevention of selenite-induced cataractogenesis. Exp Eye Res 88: 938-944, 2009

20. Bradford MM: A rapid and sensitive method for the quantitation of microgram quantities of protein utilizing the principle of protein-dye binding. Anal Biochem 72: 248-254, 1976.

21. Livak KJ and Schmittgen TD: Analysis of relative gene expression data using real-time quantitative PCR and the 2(-Delta Delta C(T)) Method. Methods 25: 402-408, 2001.

22. Rohde C, Zhang Y, Reinhardt R and Jeltsch A: BISMA - fast and accurate bisulfite sequencing data analysis of individual clones from unique and repetitive sequences. BMC Bioinformatics 11: 230, 2010.

23. Elanchezhian R, Sakthivel M, Geraldine P and Thomas PA: Regulatory effect of acetyl-l-carnitine on expression of lenticular antioxidant and apoptotic genes in selenite-induced cataract. Chem Biol Interact 184: 346-351, 2010.

24. Brennan LA and Kantorow M: Mitochondrial function and redox control in the aging eye: role of MsrA and other repair systems in cataract and macular degenerations. Exp Eye Res 88: 195-203, 2009.

25. Lou MF: Redox regulation in the lens. Prog Retin Eye Res 22: 657-682, 2003. 